\title{
Towards an Effective Design of Social Robots
}

\author{
Haizhou Li • John-John Cabibihan · Yeow Kee Tan
}

Published online: 9 November 2011

(C) Springer Science \& Business Media BV 2011

In the past decade, we have witnessed an intensive progress of technology spurred on by community initiatives. The guest editors had its first meeting in 2010 to find that it was timely for the community to collectively document the recent advances in social robotics through a special issue in the International Journal of Social Robotics. This special issue presents various designs for social robots in order to make them more effective and natural for human-robot interactions.

Social robots are autonomous robots that are able to interact and communicate among themselves, with humans, and with the environment and are designed to operate according to the established social and cultural norms. The main requirement for such robots is intelligence, which forms the basis of human-robot interaction. Many design requirements should be considered for social robots. Among these are the abilities to:

1. recognize the presence of a human through its senses such as vision, touch and sound;

2. engage in physical acknowledgment such as holding or touching;

\section{H. Li · Y.K. Tan}

Institute for Infocomm Research, Agency for Science,

Technology and Research (A*STAR), Singapore, Singapore

H. Li

e-mail: hli@i2r.a-star.edu.sg

Y.K. Tan

e-mail: yktan@i2r.a-star.edu.sg

J.-J. Cabibihan ( $\varangle)$

Social Robotics Laboratory, Interactive and Digital Media Institute; and the Department of Electrical and Computer Engineering, National University of Singapore, Singapore, Singapore

e-mail: elecjj@nus.edu.sg
3. use physical motions and gestures;

4. express and/or perceive emotions and

5. engage in a conversation.

This special issue addresses various challenges in the design of social robots, which can be used in a variety of applications, such as research platforms, toys and games, educational tools, and therapeutic aids.

Child-robot interaction has been an important topic of research towards the development of social robots for entertainment and for use as assistive tools for children who have been diagnosed with Autism Spectrum Disorders. The first article, "CHARLIE: An Adaptive Robot Design with Hand and Face Tracking for Use in Autism Therapy", by Laura Boccanfuso and Jason M. O'Kane, introduces a new design for a robot that plays interactive imitation games using hand and face tracking. The designed games were aimed at increasing attention, promoting turn-taking skills and encouraging child-led verbal and non-verbal communication through simple imitative play. In addition to the design of the adaptive robot, an algorithm to detect hand motions is presented in this paper and was evaluated through experiments with a group of typically developing children.

The second article, "Study on an Assistive Robot for Improving Imitation Skill of Children with Autism", by Isao Fujimoto, Tohru Matsumoto, P. Ravindra S. De Silva, Masakazu Kobayashi, and Masatake Higashi, investigates the effect of a humanoid robot that interacts with children with autism. To provide the robot with mimicry function and evaluate the child's motion in real time, the authors implemented the Q-Learning approach, Mixture Gaussian and Expectation-Maximization algorithms. Results from the practical experiments report an improvement in children's imitation skills.

The next paper, titled "STB: Child-Dependent Sociable Trash Box", by Yuto Yamaji, Taisuke Miyake, Yuta Yoshi- 
ike, P. Ravindra S De Silva, and Michio Okada, explores child-robot interaction and the social cues in the collection of trash by children in a child-centric environment. The proposed Social Trash Box (STB) robot uses the interactive social cues and vocal interactions to create engaging interactions with children. The approach made use of a modelbased unsupervised method to elicit the proxemic information by considering the robot's dynamic interaction with the children.

The fourth article, titled: "Maggie: A Social Robot as a Gaming Platform", by V. Gonzalez-Pacheco, Arnaud Ramey, F. Alonso-Martin, A. Castro-Gonzalez, and Miguel A. Salichs presents a social robot that offers several games that the user can play. The robot, its hardware and software architecture, and the details of the development of five games were described in this paper. The social robot, in contrast to most of the edutainment robots, is able to create new skills by using a flexible and modular architecture.

Patrick Holthaus, Karola Pitsch, and Sven Wachsmuth presented a spatial model for a humanoid robot in the fifth paper "How Can I Help? Spatial Attention Strategies for a Receptionist Robot". The authors implemented their spatial model, which is connected to an attention system to investigate the transition between distant and close communication for opening of a social interaction. The behavior that resulted from the proposed model was evaluated through an online video study, which reveals that a robot with these behaviors shows higher attention and intention compared to other existing strategies.

The sixth article, titled "GCUA Humanoid Robotic Hand with Tendon Mechanisms and its Upper Limb", by Demeng Che and Wenzeng Zhang focused on improving the capability of robotic hands to achieve humanoid manipulations with low dependence on control and sensor feedback systems. This was done through the Gesture-Changeable Under-Actuated (GCUA) grasping function. An upper limb system for a humanoid robot was designed using the robotic hand and was described.

The seventh article from Takamasa Iio, Masahiro Shiomi, Kazuhiko Shinozawa, Takaaki Akimoto, Katsunori Shimohara, and Norihiro Hagita is entitled "Investigating Entrainment of People's Pointing Gestures by Robot's Gestures Using a WOZ Method". This paper investigated the gestures that are important to the entrainment of people's pointing gestures. For this purpose, a laboratory experiment, based on a Wizard-of-Oz paradigm, was carried out in which the frequencies of a participant's pointing gestures in conversation were assessed among the three conditions of gazing, pointing, and gazing and pointing. Based on the results, it was shown that a robot's gazing gestures play an important role in the entrainment of people's pointing gestures.

The next paper, "An EEG Adaptive Information System for an Empathic Robot", a speech-driven information system is introduced for a humanoid robot by Dominic Heger,
Felix Putze, and Tanja Schultz. The strategy of the system for information presentation changes based on the brain patterns of its user. In order to evaluate the performance, brain patterns of 20 participants were classified, with a mean accuracy rate of $83.5 \%$, using EEG signals corresponding to situations of low and high mental workload. In addition, it was shown that the dynamic presentation strategy outperforms the static in both information correctness and completeness.

"Learning the Selection of Actions for an Autonomous Social Robot by Reinforcement Learning Based on Motivations" is the title of the ninth paper written by Álvaro CastroGonzález, María Malfaz, and Miguel A. Salichs. Following the previous research works focused on biologically inspired mechanisms, this paper proposes a motivational system for social robots for decision making. The proposed system was able to choose the suitable action in every state, which was determined by the dominant motivation of the robot and its relation to the present objects in its environment. This system employed reinforcement learning with the new Object Q-Learning method. Evaluation of the presented decision making system with learning process showed an improvement in the robot's performance. It is noteworthy that the evaluation is done by observing the evolution of the robot's wellbeing.

The next paper, with the title of "Multidisciplinary Design Approach for Implementation of Interactive Services", by I-Han Kuo, Chandimal Jayawardena, Elizabeth Broadbent, and Bruce A. MacDonald, discusses the critical factors of human-robot interaction for a service robot. The authors proposed a new approach to facilitate inter-disciplinary collaborations, which is essential for human-robot interaction research. The proposed approach includes vital signs measurement, medication management, and entertainment and falls detection. The method is employed in the design of the healthcare service robot "Cafero" developed at the University of Auckland in collaboration with the Electronic and Telecommunication Research Institute (ETRI) and Yujin Robot Company Ltd. of Korea. UML and UMLi are the modeling diagrams that are used to model the robot's multimodal and interactive behavior.

Last but not the least, "Visual Attention Prediction Using Saliency Determination of Scene Understanding for Social Robots", by Hongsheng He and Shuzhi Sam Ge, presents a model for the biological ability of visual attention for social robots. Visual attention assists in scene understanding and is determined by visual stimuli and prior knowledge of people's attention. The proposed model was considered for natural scenes and was evaluated using fixation data of actual eye tracking database. The results indicate the effectiveness of the approach, which determines social robots' attention in order to behave naturally.

We hope that the readers will find the articles interesting and inspiring for further research in the field. It has not 
been an easy task calling for papers and handling the reviews in such a short time. The guest editors would like to express their sincere gratitude to all contributors who made this special issue possible, and the group of reviewers who have offered their precious time and invaluable comments to improve the quality of articles. Special thanks are extended to Editor-in-Chief Professor Shuzhi Sam Ge, Ms. Nathalie Jacobs, Springer's Senior Editor for Engineering, and Mr. Anand David of the Journals Editorial Office at Springer who have assisted the guest editors from the inception to the completion of this special issue.

Guest Editors

Haizhou Li

John-John Cabibihan

Yeow Kee Tan 